\title{
Identificação das alterações metabólicas e dos parâmetros oxidativos no soro dos coletadores de lixo com e sem enteroparasitoses
}

\author{
Epidemiological profile of oxidative and metabolic waste collectors with and without \\ intestinal parasites
}

\author{
Gláucio Barros Saldanha1, Luciana Muratori Costa², Rivelilson Mendes de Freitas ${ }^{3}$, \\ ${ }^{1}$ Doutorando do Programa de Pós-Graduação em Saúde Pública da Universidad Americana; ${ }^{2}$ Bolsista de Iniciação \\ Científica -CNPq/UFPI; ${ }^{3}$ Professor Adjunto da Faculdade de Farmácia da \\ Universidade Federal do Piauí.
}

\begin{abstract}
Resumo
Introdução: Em países em desenvolvimento a prevalência elevada de enteroparasitoses afeta a qualidade de vida da população mais carente, submetida a condições precárias de higiene e cujo metabolismo, é possivelmente prejudicado. Objetivo: Identificar as alterações metabólicas e parâmetros oxidativos, e estabelecer o perfil epidemiológico da população em estudo. Metodologia: Foi realizada coleta de amostras de fezes de coletadores de lixo do município de Quixeramobim, Ceará, Brasil. Estas foram submetidas inicialmente ao parasitológico seriado, e depois os coletadores foram então submetidos à análise da função metabólica por: Hemograma Completo, Glicemia em Jejum, Ferro Sérico, Transaminase Glutâmica Pirúvica e Transaminase Glutâmica Oxalacética e por dos parâmetros do estresse oxidativo. Resultados: O perfil sócio-demográfico entre os voluntários é: homens de faixa etária predominante acima de 35 anos de idade, que se declaram brancos, com situação conjugal estável e, em média, mais de dois filhos, com vínculo empregatício definido e renda familiar de um salário mínimo. Houve diferenças apenas quanto ao tipo de moradia. $\mathrm{O}$ perfil parasitológico dos coletadores de lixo foi: monoparasitoses (Entamoeba coli, Entamoeba histolítica, Giardia lamblia, Ascaris lumbricoides e Ancilostomídeos); biparasitoses (Entamoeba histolítica e Endolimax nana ou Entamoeba coli e Entamoeba histolítica) e poliparasitoses (Giardia lamblia, Entamoeba histolítica e Endolimax nana). No perfil hematológico detectou-se redução no hematócrito e leucopenia nos coletadores com enteroparasitoses. No perfil metabólico observou-se aumento de: aspartato aminotransferase e glutâmica oxaloacética no soro do mesmo grupo. Conclusão: As enteroparasitoses podem alterar as defesas antioxidantes dos coletadores de lixo e podem estar correlacionadas com as alterações hematológicas e metabólicas.
\end{abstract}

Palavras-chave: Estresse oxidativo. Entamoeba. Giardia lamblia. Ascaris lumbricoides. Ancylostoma.

\begin{abstract}
Introduction: In developing countries the high prevalence of intestinal parasitic diseases affects life quality of the population, usually submitted to bad hygiene conditions and whose metabolism is possibly injured. Objectives: To identify the metabolic and oxidative parameters and to establish the epidemiological profile of this population. Methodology: It was collected stool samples from collectors of garbage in city of Quixeramobim Region Central Hinterland, Ceara, Brazil. The samples were subjected to series of parasitological tests. The garbage collectors were then subjected to analysis of metabolic function by means of complete blood count, fasting glucose, serum iron, serum glutamic pyruvic transaminase and aspartate aminotransferase and oxidative stress parameters. Results: The social demographic profile among the volunteers is: men predominantly above 35 years old, who declare themselves white skinned, have a stable marital status with, on average, more than two children, set with employment and family income of a minimum wage. There were differences in the type of housing. The parasitological profile of garbage collectors was: monoparasitoses (Entamoeba coli, Entamoeba histolítica, Giardia lamblia, Ascaris lumbricoides and Ancilostomídeos); biparasitoses (Entamoeba histolítica and Endolimax nana or Entamoeba coli and Entamoeba histolítica) and poliparasitoses (Giardia Lamblia, Entamoeba histolítica and Endolimax nana). The haematological profile showed low hematocrit and leucopenia in collectors with intestinal parasites. The metabolic profile analysis detected increased levels of aspartate aminotransferase and glutamic oxaloacetic in the serum of the same group. Conclusions: The intestinal parasitosis may modify the garbage collectors' antioxidant defenses and can be relacted to hematological and metabolic changes.

Keywords: Oxidative stress. Entamoeba. Giardia lamblia. Ascaris lumbricoides. Ancylostoma.
\end{abstract}

\section{INTRODUÇÃO}

A investigação de enteroparasitores entre coletadores de lixo tem sido amplamente negligenciada. É importante considerar que as campanhas de controle e erradicação das enteroparasitoses não requerem grandes financiamentos, mas necessitam de uma

Correspondência / Correspondence: Curso de Farmácia da Universidade Federal do Piauí, Campus Universitário s/n, Ininga 64.049-550, Teresina, Piauí. Tel 86-3215-5870 e-mail: rivmendes@hotmail.com imediata conscientização da população sobre os riscos inerentes às alterações metabólicas produzidas por essas doenças ${ }^{1}$.

A transmissão das enteroparasitoses está diretamente relacionada com as condições de vida e de higiene ${ }^{2}$. Uma alta prevalência de parasitoses entre coletadores de lixo pode ser verificada. No entanto, ainda não foram descritas na literatura quais as mudanças induzidas por essas doenças sobre o perfil oxidativo no soro dos coletadores de lixo. 
É importante destacar que as enteroparasitoses acarretam prejuízos incomensuráveis à qualidade de vida e à saúde dos infectados, uma vez que os afastam de suas atividades profissionais por induzirem alterações em parâmetros hematológicos e bioquímicos ${ }^{3}$. Outro ponto a ser considerado é a presença do estresse oxidativo no soro de coleratadores de lixo associado as essas alterações que precisa ser melhor investigada ${ }^{4}$.

Dessa forma, identificou-se as alterações metabólicas entre os coletadores de lixo com e sem enteroparasitoses, com o objetivo de determinar também se há uma relação entre essas alterações metabólicas como causa ou consequência do estresse oxidativo no soro de coletadores de lixo do município de Quixeramobim, Ceará.

\section{MATERIAIS E MÉTODOS}

Um estudo descritivo com características analíticas, transversal, foi realizado com a participação dos coletadores de lixo do município de Quixeramobim, Ceará, Brasil. O projeto foi aprovado pelo Comitê de Ética em Pesquisa da Faculdade Católica Rainha do Sertão (Número do Protocolo: 20090011)

Os espécimes clínicos foram coletados no período de Outubro a Dezembro de 2009. Após a coleta, realizouse o exame parasitológico de fezes em três amostras de dias alternados, e coletou-se sangue para a realização dos exames a fim de avaliar a função metabólica e parâmetros relacionados ao estresse oxidativo.

Os coletadores que aceitaram participar da pesquisa assinaram o Termo de Consentimento Livre e Esclarecido. A partir da assinatura deste, questionários semi-estruturados foram preenchidos durante entrevista de 20 a 30 minutos com os coletadores para coletar as seguintes informações: idade; sexo; endereço residencial; grau de instrução; situação habitacional; condições sócio econômicas; tipo de saneamento básico; uso ou não de equipamentos de proteção individual e o uso de medicamentos antiparasitários. Em seguida foi marcada uma segunda visita, previamente agendada, durante essa segunda etapa os coletadores levaram as três amostras de fezes colhidas em dias alternados em frasco plástico de boca larga, enroscado e fornecido pelo laboratório, contendo formol a $10 \%$.

Os participantes do estudo foram divididos em três grupos, a saber: Grupo 1: constituído por voluntários sadios sem enteroparasitoses ( $n=40$ ); Grupo 2: constituído por coletadores de lixo com enteroparasitoses $(n=30)$; Grupo 3: constituído por coletadores de lixo sem enteroparasitoses $(n=30)$. Os resultados do perfil parasitológico de fezes dos coletadores de lixo revelaram que a metade deles estava com enteroparasitoses e a outra parte da população em estudo encontrava-se sem enteroparasitoses. Por sua vez, o grupo de controle foi constituído por aqueles que não eram coletadores de lixo e não apresentavam nenhum tipo de enteroparasitose, embora apresentassem condições sócio econômicas semelhantes às dos coletadores de lixo.
Dessa forma, primeiramente as amostras coletadas foram submetidas inicialmente a exames de fezes (parasitológico de fezes seriado). No segundo momento, as amostras de sangue e soro obtidas dos coletadores de lixo foram então submetidas à análise da função metabólica por meio do Hemograma Completo pelo método automatizado da impedância elétrica, Glicemia em Jejum e Ferro Sérico. Também foram avaliados os níveis de Transaminase Glutâmica Pirúvica (TGP) e Transaminase Glutâmica Oxalacética (TGO) pelo método da cinética e adotado como valores de referências (TGO = 04 a 36U/L e TGP = 04 a $38 \mathrm{U} / \mathrm{L}$ ), bem como foram determinados no soro dos grupos em estudo os parâmetros do estresse oxidativo (níveis nitrito, conteúdo de glutationa reduzida (GSH), a concentração das substâncias reativas com o ácido tiobarbitúrico (TBARS) e a atividade da enzima antioxidante catalase).

Após a coleta das fezes as amostras foram processadas e examinadas para a determinação, ou não, da presença dos parasitas pertencentes aos grupos dos helmintos e dos protozoários ${ }^{5,6}$. Após a coleta das fezes seriada (três dias alternados) em um recipiente contendo como conservante a formalina a $10 \%(25 \mathrm{~mL}$ de formaldeído a $40 \%$ em $1 \mathrm{~L}$ de água destilada) $\left({ }^{6}\right)$, foram utilizados dois métodos de determinação, devido as dificuldades naturais para o diagnóstico das parasitoses.

O sangue coletado foi centrifugado em uma macrocentrífuga (Benfeer) e, posteriormente, separado. O plasma obtido foi utilizado para a determinação da Glicemia em Jejum e o soro para a dosagem de Ferro Sérico, TGO, TGP e dos níveis de GSH, catalase, nitrito e a concentração de TBARS. O sangue total foi usado para a determinação do Hemograma Completo.

O grau de peroxidação lipídica no soro dos três grupos investigados foi medido por meio da dosagem dos níveis de TBARS $\left({ }^{7}\right)$. A determinação do conteúdo de nitrito foi feita pelo método de Griess $\left({ }^{8}\right)$. A atividade da catalase foi avaliada pelo método previamentes descrito por Maehly e Chance $\left({ }^{9}\right)$. E a determinação da concentração da GSH foi realizada segundo a técnica previamente descrita por Sedlak e Lindsay $\left({ }^{10}\right)$. Para todos os testes a quantidade de proteína nas amostras preparadas a partir do soro foi determinada pelo método de Lowry e colaboradores ${ }^{11}$.

Todos os procedimentos realizados pelo laboratório utilizaram como controle de qualidade interno para hematologia minotrol da marca (HORIBA Medical) e para bioquímica Qualitrol da (Labtest Diagnóstica S.A.). Da mesma forma, procedeu-se o controle de qualidade externo conforme preconizado pela Sociedade Brasileira de Análises Clínicas (SBAC).

Os resultados que obedeciam a uma distribuição paramétrica foram analisados por Análise de Variância (ANOVA) com teste de Student Newman Keuls (post hoc) pelo programa GraphPad Prism versão 3.00 para Windows, GraphPad Software, San Diego California USA. Copyright (C) 1994-1999 por GraphPad software. 
Os dados não paramétricos (percentagens) foram analisados pelo mesmo programa utilizando o teste do qui-quadrado. As diferenças foram consideradas estatisticamente significativas a partir de $p<0,05$.

\section{RESULTADOS}

Analisando os dados da Tabela 1 verificou-se que o Grupo 1 (voluntários sadios sem enteroparasitoses), Grupo 2 (coletadores de lixo com enteroparasitoses) e Grupo 3 (coletadores de lixo sem enteroparasitoses) são do sexo masculino, apresentam faixa etária predominante acima de 35 anos de idade, declaram-se brancos, apresentam uma situação conjugal estável, com, em média, mais de dois filhos e com vínculo empregatício e renda familiar de um salário mínimo. Os três grupos diferem quanto ao tipo de moradia, sendo mais frequente casas alugadas para os indivíduos do grupo 1 e casas próprias para os grupos 2 e 3 (Tabela 1).

A partir da análise os dados da Tabela 2 verificouse que, entre os grupos estudados, todos os entrevistados moram em casa de tijolos com luz elétrica, possuem água encanada, mas apenas um número restrito de residências é provido de saneamento básico em suas condições ideais. Com relação ao uso de vermífugos nos últimos seis meses apenas $27,5,13,33$ e $20 \%$ dos entrevistados confirmaram o uso, nos grupos 1, 2 e 3, respectivamente.

Em relação ao uso de EPI, entre os participantes do grupo 1 (55, 40 e 5\% fazem uso de luvas, botas e máscaras, respectivamente). Já entre os grupo 2 e 3 apenas $50 \%$ faz uso apenas de luvas ou botas. Quanto ao uso do hipoclorito na água destinada ao consumo e à limpeza de alimentos, verificou-se uma baixa prevalência dessa hábito apenas no grupo 2. Por fim, foi observado que todos os entrevistados apresentam o hábito de lavar as mãos após defecar e a maioria deles em todos os grupos entrevistados lavam os alimentos crus antes da ingestão (Tabela 2).

Os resultados do perfil parasitológico de fezes dos coletadores de lixo revelou que $86,6 \%$ deles encontravamse monoparasitados: 13 com Entamoeba coli, sete com Entamoeba histolítica, dois com Giardia lamblia, dois com Ascaris lumbricoides e dois com Ancilostomídeos. Enquanto que, apenas três coletadores encontravam-se biparasitados com Entamoeba histolítica e Endolimax nana ou Entamoeba coli e Entamoeba histolítica. Apenas um coletador encontrava-se poliparasitado (Giardia lamblia, Entamoeba histolítica e Endolimax nana).

Uma diminuição significativa de $9 \%$ na quantidade de hemácias/mm3 foi evidenciada no sangue dos coletadores com enteroparasitose, quando comparado ao Grupo 1 e um aumento de 6,0 e 2,02\% no grupo 3 quando comparado aos Grupo 2 e Grupo 1 , respectivamente. Por outro lado, foi verificada uma diminuição de $19 \%$ na concentração do hematócrito do Grupo 2, quando comparado ao grupo 1 e um aumento de $25 \%$ quando comparado ao grupo 3 (Tabela 3 ).
Após a identificação da concentração de hemoglobina entre os grupos verificou-se uma diminuição significativa de $19 \%$ nos níveis de hemoglobina no Grupo 2, quando comparado ao Grupo 1. Já o Grupo 3 apresentou um aumento significativo no conteúdo da hemoglobina quando comparado ao Grupo 2.

Em relação ao número de leucócitos por mililitro foi verificada uma diminuição de $30 \%$ no Grupo 2 , quando comparado ao Grupo 1. Entretanto, quando comparados os grupos 3 e 2 foi verificado um aumento dos leucócitos de $46 \%$ no Grupo 3. Os níveis de plaquetas não demonstraram alterações significativas entre os grupos, bem como não foram observadas alterações nos parâmetros hematológicos descritos acima entre os grupos 1 e 3 (Tabela 3 ).

Uma diminuição significativa de 21 e $8 \%$ na quantidade de ferro foi evidenciada no sangue dos coletadores do Grupo 2 e do Grupo 3 quando comparados ao Grupo 1. E um aumento de $16 \%$ no Grupo 3 quando comparado ao Grupo 2 (Tabela 3).

Em relação à concentração de glicose $(\mathrm{mg} / \mathrm{dL})$ foi verificada uma diminuição de $9 \%$ no Grupo 2 quando comparado ao Grupo 1. Neste mesmo parâmetro, por sua vez, não foi verificada alteração no Grupo 3 quando comparado ao Grupo 1. Entretanto, quando comparados o grupo 3 e o grupo 2, constatou-se um aumento de $11 \%$ no grupo 3 (Tabela 3).

Nas determinações feitas sobre o nível de TGO foi verificado um aumento de $78 \%$ e uma diminuição de $15 \%$ nos grupos 2 e 3 quando comparados ao grupo 1. Por outro lado, ao comparar os resultados de TGO do Grupo 3 com os do Grupo 2 foi verificada uma redução de $52 \%$ (Tabela 3).

Naúltima análise dos resultados obtidos a partir do soro observou-se um aumento de $78 \%$ e uma diminuição de $15 \%$ nos grupos 2 e 3 quando comparados ao grupo 1 nos níveis de TGP. Todavia, ao comparar os resultados de TGP entre o Grupo 3 e o Grupo 2 foi verificada uma redução de 49,0\% (Tabela 4).

Um aumento significativo de $282 \%$ na concentração de TBARS foi evidenciado no soro dos coletadores com enteroparasitoses quando comparado ao Grupo 1 e de $267 \%$ quando comparado ao Grupo 3. Comparando-se os grupos 1 e 3 não foi observada alteração significativa no nível de TBARS. Um aumento significativo de $97 \%$ no conteúdo de nitrito foi evidenciado no soro dos coletadores com enteroparasitose quando comparado ao Grupo 1 e de $82 \%$ em relação ao Grupo 3 (Figura 1).

Um aumento significativo de $95 \%$ na atividade enzimática da catalase foi constatado no soro dos coletadores com enteroparasitoses em relação ao Grupo 1 e de $93 \%$ quando comparado ao Grupo 3. Por fim, foi observada uma diminuição significativa de $35 \%$ na concentração da GSH no soro de coletadores de lixo com enteroparasitoses quando comparado ao Grupo 1 e de $28 \%$ quando comparado ao Grupo 3. Da mesma forma, quando comparados os grupos 1 e 3 foi observada uma redução significativa no conteúdo de GSH de $10 \%$ (Figura 1). 
Tabela 1. Parte I da caracterização do perfil sócio-demográfico entre os coletadores de lixo com e sem enteroparasitoses.

\begin{tabular}{|c|c|c|c|c|c|c|}
\hline \multirow[t]{2}{*}{ Perfil sócio-econômico } & \multicolumn{2}{|c|}{ Grupo 1} & \multicolumn{2}{|c|}{ Grupo 2} & \multicolumn{2}{|c|}{ Grupo 3} \\
\hline & $n$ & $(\%)$ & $n$ & $(\%)$ & $n$ & (\%) \\
\hline \multicolumn{7}{|l|}{ Faixa etária } \\
\hline 31-35 anos & 07 & 17.5 & 05 & 16.66 & 04 & 13.33 \\
\hline$>35$ anos & 28 & 70.0 & 17 & $56.66^{\mathrm{a}}$ & 26 & 86.66 \\
\hline Total & 40 & 100 & 30 & 100 & 30 & 100 \\
\hline \multicolumn{7}{|l|}{ Cor da pele autorreferida } \\
\hline Brancos & 17 & 42.5 & 14 & $46.66^{\mathrm{a}}$ & 12 & 40.00 \\
\hline Negra & 10 & 25.0 & 08 & 26.66 & 05 & 16.66 \\
\hline Parda & 13 & 32.5 & 08 & 26.66 & 13 & $43.33^{b}$ \\
\hline Total & 40 & 100 & 30 & 100 & 30 & 100 \\
\hline \multicolumn{7}{|l|}{ Estado Civil } \\
\hline Solteiro & 13 & 32.5 & 08 & 26.66 & 10 & 33.33 \\
\hline Casado & 27 & 67.5 & 22 & $73.33^{\mathrm{a}}$ & 20 & $66.66^{\mathrm{b}}$ \\
\hline Total & 40 & 100 & 30 & 100 & 30 & 100 \\
\hline \multicolumn{7}{|l|}{ Número de filhos } \\
\hline 0 & 12 & 30.0 & 06 & 20 & 05 & 16.66 \\
\hline 1 & 05 & 12.5 & 04 & 13.33 & 02 & 6.66 \\
\hline 2 & 09 & 22.5 & 08 & 26.66 & 13 & $43.33^{b}$ \\
\hline$>2$ & 14 & 35.0 & 12 & $40^{a}$ & 10 & 33.33 \\
\hline Total & 40 & 100 & 30 & 100 & 30 & 100 \\
\hline \multicolumn{7}{|l|}{ Renda Familiar } \\
\hline 1 salário & 40 & 100 & 30 & $100^{\mathrm{a}}$ & 26 & $86.66^{b}$ \\
\hline 1-2 salários & 00 & 00 & 00 & 00 & 02 & 6.66 \\
\hline 2-4 salários & 00 & 00 & 00 & 00 & 01 & 3.33 \\
\hline Total & 40 & 100 & 30 & 100 & 30 & 100 \\
\hline \multicolumn{7}{|l|}{ Sexo } \\
\hline Masculino & 31 & 77.5 & 30 & $100^{\mathrm{a}}$ & 28 & $93.33^{b}$ \\
\hline Feminino & 09 & 22.5 & 00 & 00 & 02 & 6.66 \\
\hline Total & 40 & 100 & 30 & 100 & 30 & 100 \\
\hline \multicolumn{7}{|l|}{ Vinculo empregatício } \\
\hline Sim & 33 & 82.5 & 30 & $100^{\mathrm{a}}$ & 29 & $96.66^{b}$ \\
\hline Não & 07 & 17.5 & 00 & 00 & 01 & 3.33 \\
\hline Total & 40 & 100 & 30 & 100 & 30 & 100 \\
\hline \multicolumn{7}{|l|}{ Tipo de moradia } \\
\hline Própria & 18 & 45.0 & 16 & $53.33^{\mathrm{a}}$ & 20 & $66.66^{b}$ \\
\hline Alugada & 19 & 47.5 & 10 & 33.33 & 08 & 26.66 \\
\hline Cedida & 03 & 7.5 & 04 & 13.33 & 02 & 6.66 \\
\hline Total & 40 & 100 & 30 & 100 & 30 & 100 \\
\hline
\end{tabular}

Grupo 1: Foi constituído por voluntários sadios sem enteroparasitoses ( $\eta=40)$. Grupo 2: Foi constituído por coletadores de lixo com enteroparasitoses $(\eta=30)$. Grupo 3: Foi constituído por coletadores de lixo sem enteroparasitoses $(\eta=30)$. Os valores representam a percentagem (\%) do número de pacientes entre parênteses. ${ }^{a} p<0,01$ quando comparado ao Grupo $1 ;{ }^{b} p<0,01$ quando comparado ao Grupo 2. 
Tabela 2. Parte II da caracterização do perfil sócio-demográfico entre os coletadores de lixo com e sem enteroparasitores.

\begin{tabular}{|c|c|c|c|c|c|c|}
\hline \multirow[t]{2}{*}{ Perfil sócio-econômico } & \multicolumn{2}{|c|}{ Grupo 1} & \multicolumn{2}{|c|}{ Grupo 2} & \multicolumn{2}{|c|}{ Grupo 3} \\
\hline & $\mathbf{n}$ & $(\%)$ & $n$ & $(\%)$ & $\mathrm{n}$ & $(\%)$ \\
\hline \multicolumn{7}{|l|}{ Espécie de moradia } \\
\hline Alvenaria & 00 & 00 & 21 & $70^{\mathrm{a}}$ & 24 & 80 \\
\hline Madeira & 00 & 00 & 02 & 6.66 & 00 & 00 \\
\hline Tijolo & 40 & 100 & 05 & 23.32 & 06 & $19.99^{b}$ \\
\hline \multicolumn{7}{|l|}{ Água } \\
\hline Encanada & 31 & 77.5 & 26 & $86.66^{\mathrm{a}}$ & 28 & $93.33^{\mathrm{b}}$ \\
\hline Poço & 09 & 22.5 & 04 & 13.33 & 02 & 6.66 \\
\hline Total & 40 & 100 & 30 & 100 & 30 & 100 \\
\hline \multicolumn{7}{|l|}{ Saneamento } \\
\hline Esgoto & 05 & 12.5 & 04 & 13.33 & 08 & 26.66 \\
\hline Fossa & 21 & 52.5 & 05 & 16.66 & 06 & 20 \\
\hline Céu aberto & 14 & 35.0 & 21 & $70^{a}$ & 16 & $53.33^{b}$ \\
\hline Total & 40 & 100 & 30 & 100 & 30 & 100 \\
\hline \multicolumn{7}{|l|}{ Luz elétrica } \\
\hline Sim & 40 & 100 & 30 & $100^{\mathrm{a}}$ & 30 & 100 \\
\hline Não & 00 & 00 & 00 & 00 & 00 & 00 \\
\hline Total & 40 & 100 & 30 & 100 & 30 & 100 \\
\hline \multicolumn{7}{|c|}{ Fez uso de medicação para verme } \\
\hline Sim & 11 & 27.5 & 04 & 13.33 & 06 & 20 \\
\hline Não & 29 & 72.5 & 26 & $86.66^{a}$ & 24 & $80^{b}$ \\
\hline Total & 40 & 100 & 30 & 100 & 30 & 100 \\
\hline \multicolumn{7}{|l|}{ Faz uso de EPI's } \\
\hline Luvas & 22 & 55 & 15 & 50 & 15 & 50 \\
\hline Botas & 16 & 40 & 15 & 50 & 15 & 50 \\
\hline Máscara & 02 & 05 & 00 & 00 & 00 & 00 \\
\hline Total & 40 & 100 & 30 & 100 & 30 & 100 \\
\hline \multicolumn{7}{|l|}{ Ao defecar lava as mãos } \\
\hline Sim & 40 & 100 & 30 & $100^{\mathrm{a}}$ & 30 & 100 \\
\hline Não & 00 & 00 & 00 & 00 & 00 & 00 \\
\hline Total & 40 & 100 & 30 & 100 & 30 & 100 \\
\hline \multicolumn{7}{|l|}{ Lava os alimentos crus } \\
\hline Sim & 37 & 92.5 & 26 & $86.66^{a}$ & 29 & $96.66^{b}$ \\
\hline Não & 03 & 7.5 & 04 & 13.33 & 01 & 3.33 \\
\hline Total & 40 & 100 & 30 & 100 & 30 & 100 \\
\hline \multicolumn{7}{|l|}{ Utiliza hipoclorito } \\
\hline Sim & 30 & 75 & 08 & 26.66 & 23 & $76.66^{b}$ \\
\hline Não & 10 & 25 & 22 & $73.33^{a}$ & 07 & 23.33 \\
\hline Total & 40 & 100 & 30 & 100 & 30 & 100 \\
\hline
\end{tabular}

Grupo 1: Foi constituído por voluntários sadios sem enteroparasitoses $(\eta=40)$. Grupo 2: Foi constituído por coletadores de lixo com enteroparasitoses $(\eta=30)$. Grupo 3: Foi constituído por coletadores de lixo sem enteroparasitoses $(\eta=30)$. Os valores representam a percentagem (\%) do número de pacientes entre parênteses. Para análise estatística foi usado o teste do $\chi 2$ (qui-quadrado). ${ }^{a} p<0,01$ quando comparado ao Grupo $1 ;{ }^{b} p<0,01$ quando comparado ao Grupo 2. 
Tabela 3. Resultados do hemograma completo do sangue obtido entre os coletadores de lixo com e sem enteroparasitoses.

\begin{tabular}{|c|c|c|c|}
\hline \multirow[t]{2}{*}{ Parâmetros } & Grupo 1 & Grupo 2 & Grupo 3 \\
\hline & Média \pm S.E.M & Média \pm S.E.M & Média \pm S.E.M \\
\hline \multicolumn{4}{|l|}{ Hematológicos } \\
\hline Hemácias $\left(/ \mathrm{mm}^{3}\right)$ & $5.01 \pm 39.5$ & $4.34 \pm 63.7^{\mathrm{a}}$ & $5.31 \pm 60.20^{b}$ \\
\hline Hemoglobina $(\mathrm{g} / \mathrm{dL})$ & $14.2 \pm 0.52$ & $11.5 \pm 0.22^{\mathrm{a}}$ & $14.3 \pm 0.32^{b}$ \\
\hline Hematócrito (\%) & $45.46 \pm 1.95$ & $36.67 \pm 3.57^{\mathrm{a}}$ & $45.91 \pm 2.26^{b}$ \\
\hline Leucócitos $(/ \mathrm{mL})$ & $7.65 \pm 1.09$ & $5.36 \pm 1.51^{\mathrm{a}}$ & $7.82 \pm 1.49^{b}$ \\
\hline Plaquetas $\left(/ \mathrm{mm}^{3}\right)$ & $256.45 \pm 38.47$ & $255.06 \pm 80.51$ & $257.1 \pm 69.27$ \\
\hline \multicolumn{4}{|l|}{ Bioquímicos } \\
\hline Ferro $(\mathrm{mg} / \mathrm{dL})$ & $103.0 \pm 11.75$ & $81.70 \pm 11.29^{\mathrm{a}}$ & $94.37 \pm 11.09^{\mathrm{a}, \mathrm{b}}$ \\
\hline Glicose (mg/dL) & $80.77 \pm 6.19$ & $73.43 \pm 4.07^{\mathrm{a}}$ & $81.53 \pm 8.03^{b}$ \\
\hline $\mathrm{TGO}(\mathrm{U} / \mathrm{L})$ & $14.90 \pm 3.07$ & $26.47 \pm 5.64^{a}$ & $12.67 \pm 4.14^{\mathrm{a}, \mathrm{b}}$ \\
\hline $\operatorname{TGP}(\mathrm{U} / \mathrm{L})$ & $10.57 \pm 4.17$ & $29.53 \pm 6.08^{a}$ & $15.17 \pm 6.082^{a, b}$ \\
\hline
\end{tabular}

Grupo 1. Foi constituído por voluntários sadios sem enteroparasitoses $(\eta=40)$. Grupo 2: Foi constituído por coletadores de lixo com enteroparasitoses $(\eta=30)$. Grupo 3: Foi constituído por coletadores de lixo sem enteroparasitoses $(\eta=30)$. Os valores representam a média \pm E.P.M. do número de pacientes entre parênteses. Para análise estatística foi usado ANOVA e teste t-StudentNeuman-Keuls como post-hoc teste. ${ }^{a} p<0,01$ quando comparado ao Grupo 1 (ANOVA e teste t-Student-Neuman-Keuls como post-hoc teste); ${ }^{b} p<0,01$ quando comparado ao Grupo 2 (ANOVA e teste t-Student-Neuman-Keuls como post-hoc.
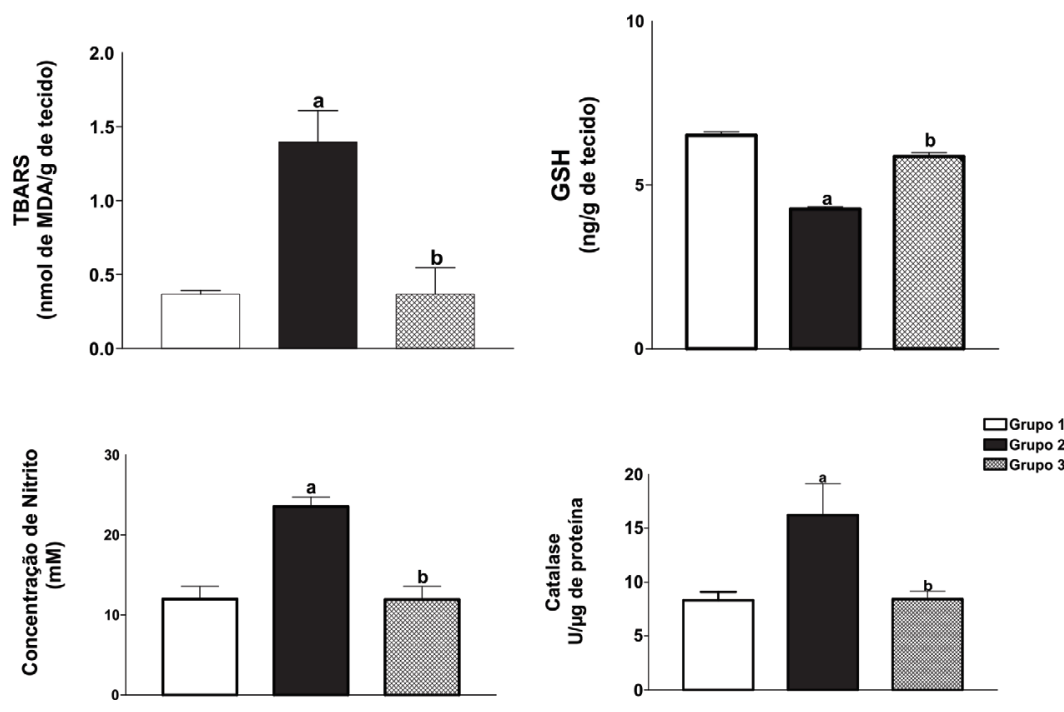

Figura 1. Determinação do conteúdo de substâncias reativas com o ácido tiobarbitúrico (TBARS), conteúdo de nitrito, concentração de glutationa reduzida (GSH) e atividade da catalase no soro de coletadores de lixo. Os resultados da verificação da produção de TBARS, concentração de nitrito, atividade da catalase e conteúdo de GSH foram expressos em nmol de malonaldeído $(\mathrm{MDA}) / \mathrm{g}, \mathrm{mM}, \mathrm{U} / \mu \mathrm{g}$ de proteína e ng/g de tecido, respectivamente. Grupo 1: Foi constituído por voluntários sadios sem enteroparasitoses $(n=40)$. Grupo 2: Foi constituído por coletadores de lixo com enteroparasitoses $(n=30)$. Grupo 3: Foi constituído por coletadores de lixo sem enteroparasitoses $(\eta=30)$. Os valores representam a média \pm E.P.M. do número de pacientes entre parênteses. ${ }^{a} p<0,001$ e ${ }^{b} p<0,001$ (ANOVA e teste $t$-Student-Neuman-Keuls como post-hoc teste). 


\section{DISCUSSÃO}

A primeira parte da caracterização do perfil sóciodemográfico entre os coletadores de lixo com e sem enteroparasitoses corroboram com os encontrados em outros estudos epidemiológicos realizados em outros estados brasileiros ${ }^{12-14}$.

Na segunda fase da caracterização do perfil sócio demográfico é importante destacar que os resultados sobre o uso de vermífugos nos últimos 6 meses revelou que apenas um pequeno número de participantes do grupo faz uso dos mesmo, e esse mesmo grupo foi o que apresentou a maior presença de enteroparasitos. Também é importante considerar que em todos os grupos entrevistados é comum o hábito de lavar as mãos após defecar e de lavar os alimentos crus antes da ingestão, embora o grupo de coletadores tenha apresentado uma alta prevalência de infestações por helmintos e protozoários. Os achados deste trabalho corroboram os encontrados em outros estudos realizados em outros estados brasileiros $\left({ }^{14}\right)$.

Estudos anteriores demonstram que há a necessidade dos coletadores de lixo utilizarem equipamentos de proteção individual. Mesmo com essas medidas de proteção foi verificado que há um grande número de acidentes de trabalho entre os coletadores ${ }^{15}$. Nesse mesmo estudo podemos observar que são oferecidos serviços médicos a eles, porém apenas $75 \%$ dos participantes da pesquisa afirmaram usufruir desse direito. No município em estudo não conseguimos obter essa informação, no entanto registramos entre as respostas dos entrevistados que a maioria dos nossos participantes relataram ter dificuldade no acesso aos serviços de saúde do Sistema Único de Saúde (SUS).

O parasitismo intestinal ainda constitui um dos mais sérios problemas de saúde pública no Brasil. Em comunidades vulneráveis das periferias, mais de $50 \%$ dos parasitológicos de fezes são positivos para um ou múltiplos parasitas ${ }^{16}$. Conforme a Organização PanAmericana de $\mathrm{Saúde}^{17}$, as geohelmintoses são altamente frequentes na América Latina, com prevalência estimada de $30 \%{ }^{18}$. A população estudada no presente trabalho apresenta alto risco de contrair a infestação ou tornar-se infestada a qualquer momento.

As enteroparasitoses são endêmicas na população investigada, como demonstrado neste estudo. Os fatores epidemiológicos da infecção entre coletadores de lixo são similares aos de outras populações de baixo nível sócio-econômico ${ }^{19}$. Dessa forma, finalizando nossas observações sobre o perfil parasitológico entre os coletadores, houve sete participantes com E. histolytica identificada. O diagnóstico diferencial entre E. histolytica e E. dispar não pode ser feito pelo simples exame microscópico, mesmo se identificados trofozoitas com hemácias deglutidas. O diagnóstico de E. histolytica requer a identificação de antígenos ${ }^{20}$, o que não foi possível ser feito em nosso estudo.

As demais amebas identificadas (E. nana e E. coli) não causam qualquer transtorno ao coletador de lixo, além de eventualmente serem confundidas com a E. histolytica e levarem a um tratamento farmacológico desnecessário. No entanto, não há informação sobre eventuais benefícios aos coletadores de lixo quando da convivência com esses protozoários e justificam a necessidade da realização do estudo.

Os resultados obtidos demonstram alterações significativas em parâmetros hematológicos e sugerem que o aumento na sobrecarga de exercício físico diminui a síntese de glutamina ${ }^{21}$, justificando os resultados encontrados com relação às alterações metabólicas e hematológicas em nosso estudo.

Além dos fatores fisiológicos, nutricionais e infecciosos, devem ser lembrados como agentes agravantes, e muitas vezes determinantes da deficiência de ferro, o baixo nível sócio-econômico e cultural e as condições de saneamento básico. Os achados também demonstram níveis de baixos a moderados para as enzimas (TGP e TGO) que podem ser justificados pela presença de infecções parasitárias. Além disso, outras mudanças sem importância clínica também foram observadas no número de leucócitos e plaquetas.

A peroxidação lipídica produzida em muitas condições patológicas, tem sido avaliada e pode ser verificada indiretamente por meio do soro e em diferentes tecidos ${ }^{22}$. A peroxidação lipídica é um processo que envolve a oxidação de componentes básicos de membranas biológicas. Os compostos resultantes da peroxidação lipídica reagem principalmente com o DNA mostrando ação genotóxica e mutagênica ${ }^{23}$, o que pode estar acontecendo no soro dos participantes do estudo.

Os resultados encontrados demonstraram que durante enteroparasitores ocorre um aumento significativo na produção de lipídios peróxidos no soro dos coletadores. Entretanto, as bases químicas com relação à produção da peroxidação lipídica e outros aspectos relacionados as enteroparasitoses, ainda permanecem pouco esclarecidos.

Neste estudo, uma variedade de processos bioquímicos incluindo a ativação de fosfolipases de membrana, proteases e nucleases podem ocorrer, contudo, como e quando estes processos se iniciam e são propagados ainda precisam ser esclarecidos entre os coletadores de lixo com enteroparasitoses.

As alterações no metabolismo dos fosfolipídios de membrana que estão envolvidas em inúmeras condições patológicas, indiretamente, parecem refletir nos mecanismos do estresse oxidativo ${ }^{24}$. Os dados "in vivo" podem confirmar a hipótese de que a peroxidação lipídica no soro produzida pelo ataque de radicais livres pode outros causar danos hematológicos e participa do estabelecimento da fisiopatologia das enteroparasitoses.

No modelo proposto para o estudo, pode ocorrer uma grande participação de dano na membrana celular em diferentes órgãos e sistemas. De um modo geral, o estresse oxidativo pode ser um dos indutores dessas 
lesões. As espécies reativas derivadas do oxigênio (ERO) quando são produzidas em excesso, podem causar dano tecidual, incluindo peroxidação lipídica, dano ao DNA, inativação de enzimas e morte celular ${ }^{25}$.

As ERO produzidas durante as enteroparsitoses podem ser consideradas como uma parte dos mecanismos envolvidos na excitotoxicidade in vivo. Acredita-se que o aumento no influxo intracelular de cálcio pode estimular a formação de ERO através de vários mecanismos levando ao consequente aumento de nitrito e nitrato ${ }^{26}$. Durante as enteroparasitoses pode-se sugerir que a formação de nitrito tem sido implicada em muitos dos mecanismos moleculares do processo, podendo modular uma cascata de efeitos citotóxico em parâmetros hematológicos.

A possível relação de causa entre a produção de ERO e a fisiopatologia das enteroparasitoses tem sido muito mais proposta do que demonstrada. As enteroparasitoses produzem uma série de mudanças em parâmetros relacionados com a produção e eliminação de ERO. Um aumento nessa formação pode ser acompanhado por um mecanismo compensatório de aumento na atividade das enzimas removedoras de radicais livres, e este aumento pode estar relacionado como um mecanismo compensatório em função dos parasitas, por meio da modulação da atividade das enzimas envolvidas no metabolismo dos radicais livres.

A glutationa reduzida (GSH, L- $\psi$-glutamil-Lcisteinil-glicina) pode ser considerada um dos agentes mais importantes do sistema de defesa antioxidante $\left({ }^{27}\right)$. Os efeitos das enteropasitoses em nível de sistema de defesa antioxidante mediado pela GSH, nos experimentos "in vitro", revelaram que no processo parasitário a interferência no conteúdo de GSH ocorre de forma aguda e que podem apresentar uma longa duração como visto pela redução no seu conteúdo após a coleta do sangue e a preparação do soro a fim de proteger contra danos celulares extensos e diminuir a gravidade das infecções parasitárias. Os resultados concordam com os dados obtidos por outros estudos ${ }^{28}$, com relação à presença de peroxidação lipídica e a redução no conteúdo de GSH.

Com relação ao efeito antioxidante protetor mediado pelo GSH, é possível sua generalização quanto ao padrão de agressão e defesa celular antioxidante para grande parte dos tecidos do organismo humano, além do soro investigado, sendo de fundamental importância para o funcionamento normal do organismo, já que o tecido hematopoiético é um dos tecidos mais vulneráveis ao estresse oxidativo por agentes exógenos ou endógenos.

\section{CONCLUSÃO}

Com base nos estudos do perfil metabólico detectou-se um aumento dos níveis das transaminases (aspartato aminotransferase e glutâmica oxaloacética) no soro dos coletadores de lixo com enteroparasitoses. Os dados coletados podem confirmar a hipótese de que há um aumento da peroxidação lipídica, na produção de nitrito e da atividade enzimática da catalase no soro, bem como as enteroparasitoses podem alterar as defesas antioxidantes, entre elas catalase e os níveis GSH no soro dos coletadores de lixo que podem estar correlacionadas direta ou indiretamente com as alterações hematológicas e metabólicas observadas entre os coletadores de lixo.

\section{REFERÊNCIAS}

1. DE CARLI, G.A.; et al. Prevalência das enteroparasitoses na população urbana e rural da região carbonífera da cidade de Arroio dos Ratos, Rio Grande do Sul. Rev. Bras. Farm., Rio de Janeiro, v. 78, p. 83-85, 1997.

2. DE CARLI, G.A.; CANDIA E. Prevalência de geohelmintos entre escolares residentes nas vilas periféricas de Porto Alegre, RS. Rev. Bras. Farm., Rio de Janeiro, v.73, n. 1, p. 7-8, 1992.

3. COSTA, M.S.G.; NUNES, M.P.P.; NUNES, J.F.L. Ocorrências de parasitoses intestinais em escolares pertencentes ao grupo escolar Vilagram Cabrita, Natal-RN. Rev. Saúde, Natal, v. 5, p. 43-46, 1990.

4. KEHER, J.P. Free radicals as mediators of tissue injury and disease. Crit. Rev. Toxicol., Boca Raton, v. 23, n. 1, , p. 21-48, 1993.

5. HOFFMANN, W.A.; PONS, J.A.; JANER, J.L. The sedimentationconcentration method in Schistosomiasis mansoni. J. Publ. Health, Oxford, v. 9, p. 281-298, 1934.

6. CARLI, G.A. Parasitologia clínica: seleção de métodos e técnicas de laboratório para o diagnóstico das parasitoses humanas. 2. ed., São Paulo: Atheneu, 2007, 906p.

7. DRAPER, H.H.; HADLEY, M. Malondialdehyde determination as an index of lipid peroxidation. Methods. Enzymol., New York, v. 186, p. 421-431, 1990

8. GREEN, L.C.; TANNENBAUM, S.R.; GOLDMAN, P. Nitrate synthesis in the germfree and conventional rat. Science, Washington, v. 212, n. 4490, p. 56-58, 1981.

9. MAEHLY, A.C.; CHANCE, B. The assay catalase and peroxidases. Methods Biochem. Anal., New York, v. 1, p. 357-359, 1954.

10. SEDLAK, J.; LINDSAY, R.H. Estimation of total protein bound and nonprotein sulfhydril groups in tissues with Ellman's reagent. Anal. Biochem., New York, v. 25, p. 192-205, 1968.

11. LOWRY, O.H..et al. Protein measurement with follin phenol reagent. J. Biol. Chem., Baltimore, v. 193, n.1, p. 265-275, 1951.

12. OLIVEIRA, C.P.A.; FREITAS, R.M. Projective instrument for the deployment of pharmaceutical care to patients with psychosocial disorders attended by the psychosocial care center. SMAD, Rev. Eletrônica Saúde Mental Álcool Drog., Ribeirão Preto, v. 4, n. 2, p. 1-15, 2008.

13. RODRIGUES, J.S.M.; FERREIRA, N.M.L.A. Caracterización del perfil epidemiológico del cáncer en una ciudad al interior de São Paulo: el conocimiento para la acción. Rev. Bras. Cancerol., Rio de Janeiro, v. 56, n. 4, p. 431-441, 2010.

14. CASADO, L.; VIANNA, L.M.; THULER, L.C.S. Factores de riesgo para enfermedades crônicas no transmissibles em Brasil: uma revisión sistemática. Rev. Bras. Cancerol., Rio de Janeiro, v.55, n. 4, p. 379-388, 2009.

15. CAMILO, R.N. et al. Perfil epidemiológico e ocupacional dos coletores de lixo de uma empresa terceirizada do município de Belém - PA. In: SEMANA DE EXTENSÃO E I JORNADA DE PESQUISA, 2., 2009, Belem Para. 
16. FERREIRA, M.U.; FERREIRA, C.S.; MONTEIRO, C.A. Tendência secular das parasitoses intestinais na infância na cidade de São Paulo (19841996). Rev. Saude Publica, São Paulo, v. 34, suppl. 6, p. 73-82, 2000

17. SILVA, S.S. Situación de salud de los pueblos indígenas de Perú. Washington: Organización Panamericana de la Salud., 1998. p 38.

18. KORKES, F.; et al. Relationship between intestinal parasitic infection in children and soil contamination in an urban slum. J. Trop. Pediatr., London, v. 55, n. 1, p. 42-45, 2009.

19. ROBINSON, L.G.; et al. Helicobacter pylori prevalence among indigenous peoples of South America. J. Infect. Dis., Chicago, v. 186, n. 8, , p. 1131-1137, 2002.

20. FOTEDAR, R.; Laboratory diagnostic techniques for Entamoeba species. Clin. Microbiol. Rev., Washington, v. 20, n. 3, p. 511-532, 2007. 21. MARKELL, E.K.; JOHN, D.T.; KROTOSKI, W.A. Parasitologia Médica. 8. ed. Rio de Janeiro: Guanabara Koogan; 2003, 501p.

22. HISCOCK N., Pedersen B.K. Exercise-induced immunodepression Plasma glutamine is not the link. J. Appl. Physiol., Washington, v. 93, n. 3, p. 813-822, 2002.

23. BRUCE A.J., BAUDRY M. Oxygen free radicals in rat limbic structures after kainate-induces seizures. Free Radic. Biol. Med., New York, v. 18, n. 6, p. 993-1002, 1996.
24. FREITAS, R.M. et al. Pilocarpine-induced seizures in adult rats: lipid peroxidation level, nitrite formation, GABAergic and glutamatergic receptor alterations in the hippocampus, striatum and frontal cortex. Pharmacol. Biochem. Behav., Phoenix, v. 78, n. 2, p. 327-332, 2004.

25. LUCZAJ, W.; SKRZYDLEWSKA, E. The compounds resulting from lipid peroxidation mostly react with DNA showing both genotoxic and mutagenic action; among them, 4-hydroxynonenal is the most genotoxic, while MDA is the most mutagenic DNA damage caused by lipid peroxidation products. Cell. Mol. Biol. Lett., Wroclaw, v. 8, n. 2, p. 391-410, 2003

26. SIESJO, B.K.; WIELOCH, T. Epileptic brain damage: pathophysiology and neurochemical pathology. Adv. Neurol., New York, v. 44, p. 813847, 1986.

27. PATEL M., LIANG, L. Mitochondrial oxidative stress and increased seizure susceptibility in SOD2-mice. Free Rad. Biol. Med., lowa, v. 36, n. 5, p. 542-554, 2004.

28. LEES, G.J. Inhibition of sodium-potassium-ATPase: potentially ubiquitous mechanism contributing to central nervous system neuropathology. Brain Res., Amsterdam, v. 16, n. 3, p. 283-300, 1991.

Submetido em 15/12/2012;

Aceito em 02/04/2013. 